Si mul at i on of magnet i zat i on process of Pur e- type super conduct or nagnet undul at or based on T- net hod

\begin{tabular}{|l|l|}
\hline 著者 & $\begin{array}{l}\text { DERI Yi , KAWAGUCH Hi deki , TSUCH MOTO } \\
\text { Nasanor i , TANAKA Takashi }\end{array}$ \\
\hline $\begin{array}{l}\text { j our nal or } \\
\text { publ i cat i on t i t l e }\end{array}$ & $\begin{array}{l}\text { Physi ca C: Super conduct i vi ty and i t s } \\
\text { Appl i cat i ons }\end{array}$ \\
\hline vol une & 518 \\
\hline page r ange & $106-110$ \\
\hline year & 2015-11-15 \\
\hline URL & ht t p: //hdl . handl e. net /10258/00008880 \\
\hline
\end{tabular}


Si mul at i on of magnet i zat i on process of Pur e- type super conduct or nagnet undul at or based on T- net hod

\begin{tabular}{|l|l|}
\hline 著者 & $\begin{array}{l}\text { DERI Yi , KAWAGUCH H deki , TSUCH MOTO } \\
\text { Nasanor i , TANAKA Takashi }\end{array}$ \\
\hline $\begin{array}{l}\text { j our nal or } \\
\text { publ i cat i on t i t l e }\end{array}$ & $\begin{array}{l}\text { Physi ca C: Super conduct i vi ty and i t s } \\
\text { Appl i cat i ons }\end{array}$ \\
\hline vol une & 518 \\
\hline page r ange & $106-110$ \\
\hline year & $2015-11-15$ \\
\hline URL & ht t p: //hdl . handl e. net /10258/00008880 \\
\hline
\end{tabular}




\title{
Simulation of Magnetization Process of Pure-type Superconductor Magnet Undulator Based on T-method
}

\author{
Yi Deri ${ }^{\mathrm{a}}$, Hideki Kawaguchi ${ }^{\mathrm{a},}{ }^{*}$, Masanori Tsuchimoto ${ }^{\mathrm{b}}$, Takashi Tanaka ${ }^{\mathrm{c}}$

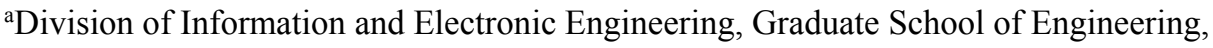 \\ Murorann Institute of Technology, 27-1 Mizumoto-cho, Muroran, Hokkaido, 050-8585, Japan \\ ${ }^{b}$ Department of Electrical and Electronic, Hokkaido University of Science, \\ 7-15-4-1 Maeda, Teine, Sapporo, Hokkaido, 006-8585, Japan \\ 'RIKEN SPring-8 Center, Koto 1-1-1, Sayo, Hyogo 679-5148, Japan \\ “kawa@mmm.muroran-it.ac.jp
}

Received:; Accepted:; Published:

\begin{abstract}
For the next generation Free Electron Laser, Pure-type undulator made of high Tc superconductors (HTSs) was considered to achieve a small size and high intensity magnetic field undulator. In general, it is very difficult to adjust the undulator magnet alignment after the HTS magnetization since the entire undulator is installed inside a cryostat. The appropriate HTS alignment has to be determined in the design stage. This paper presents the development of a numerical simulation code for magnetization process of the Pure-type HTS undulator to assist the design of the optimal size and alignment of the HTS magnets.
\end{abstract}

Keywords: Free Electron Laser (FEL); High Tc Superconductor (HTS); current vector potential method (T-method); critical state model

\section{Introduction}

As one of challenging technologies in the next generation Free Electron Laser (FEL), application of high-Tc superconductors (HTS) for undulator magnets has been proposed to obtain very strong magnetic field [1-3]. Since the HTSs for the undulator have to be magnetized all together in a cryostat, it is not easy to create sinusoidal magnetic field along the electron trajectory. The "Pure-type" HTS undulator was proposed as one of possible HTS magnet arrays for undulators [2]. In the design of the HTS undulator, it is very important to understand 
Physica $C$

and to predict the magnetization process of the HTSs. The appropriate size and alignment of the HTS undulator magnets are determined to create the exactly sinusoidal magnetic field. The numerical simulation of the magnetization process plays a very important role to design the HTS magnet.

This paper presents a numerical simulation of the magnetization process of the Pure-type HTS undulator based on the current vector potential method (T-method), combining with the critical state model for the shielding current in the HTS. The simulation results are compared with experimental measurements of the trapped fields in the HTS. Appropriate values of critical current and external magnetic field are considered by using the developed code. A simulation of a single electron motion is shown to confirm the calculated undulator magnetic field.

\section{Pure-type superconducting undulator}

Figure 1(a) shows an overview of the Pure-type HTS undulator made of three HTSs [2]. In the field-cooled magnetization process, external magnetic field is first applied to the HTS, and the HTS is cooled to be the superconducting state. During the external magnet field is gradually reduced to $B_{\min }<0$, as the bias field (Fig. 1(b)), shielding currents are induced and the field is trapped in the HTS as shown in Fig. 1(c). The alternating vertical magnetic field is created on the HTS by these shielding currents. The FEL undulator magnetic field is composed by superposition of the shielding current magnetic field and the externally bias field.

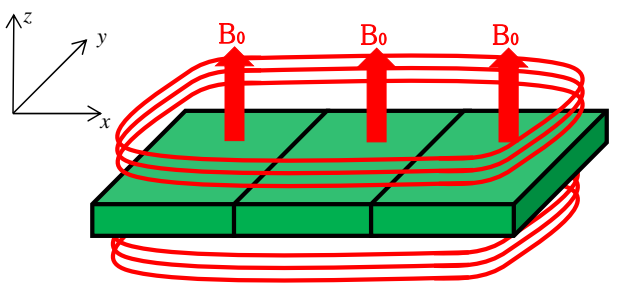

(a)

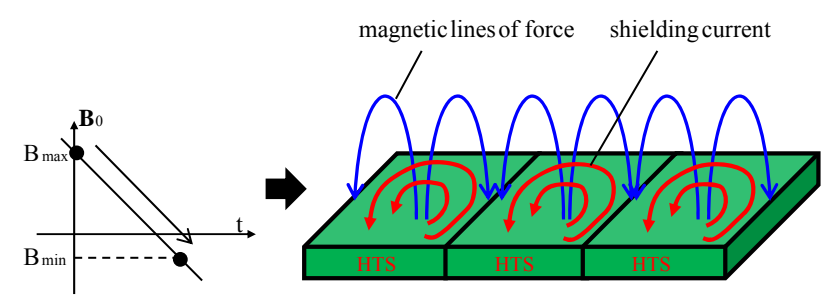

(b) (c)

Fig. 1. Overview of Pure-type undulator

\section{Simulation of magnetization process of Pure-type HTS undulator based on T-method}

The numerical simulation has very important role to understand the HTS magnetization process and the design of the undulator magnet array. In this work, we here use the current vector potential method (T-method) [4-7] for the simulation of the induced shielding current 
combing with the critical state model. The shielding current $\mathbf{J}$ induced in the HTS is expressed by using the current vector potential $\mathbf{T}$ defined by $\mathbf{J}=\nabla \times \mathbf{T}$ and the governing equation for $\mathbf{T}$ is the following integro-differential equation,

$$
\nabla \times \frac{1}{\sigma} \nabla \times \mathbf{T}-\mu_{0} \frac{\partial \mathbf{T}}{\partial \mathrm{t}}-\frac{\mu_{0}}{4 \pi} \int \frac{\partial \mathbf{T} \cdot \mathbf{n}}{\partial \mathrm{t}} \nabla^{\prime}\left(\frac{1}{\left|\mathbf{r}-\mathbf{r}^{\prime}\right|}\right) \mathrm{d} \mathrm{S}^{\prime}=\frac{\partial \mathbf{B}_{0}}{\partial \mathrm{t}},
$$

where $\sigma$ is conductivity, $\mu_{0}$ is permeability, $\mathrm{S}$ is surface of the HTS, $\mathbf{n}$ is a unit normal vector on $\mathrm{S}$, and $\mathbf{B}_{0}$ is externally applied magnetic field. The current vector potential has to satisfy the following boundary and gauge conditions respectively,

$$
\begin{aligned}
& \mathbf{T} \times \mathbf{n}=0 \quad \text { on } \mathrm{S}, \\
& \nabla \cdot \mathbf{T}=0 \quad \text { in domain. }
\end{aligned}
$$

We here assume that the shielding current is induced in $x-y$ horizontal plane, since the bulk HTS has anisotropic critical current. Therefore, the HTS can be expressed by the thin-plate multi-layers model (Fig. 2), and the current vector potential has only z-component. Ohm's law is modified to the following critical state model [4-6] for describing the shielding current behavior in the HTS,

$$
\begin{cases}\mathbf{J}=\mathrm{Jc} \frac{\mathbf{E}}{|\mathbf{E}|} & \text { if }|\mathbf{E}| \neq 0 \\ \frac{\partial \mathbf{J}}{\partial \mathrm{t}}=0 & \text { if }|\mathbf{E}|=0\end{cases}
$$

When the electric field $E$ is induced in a local region by change of the magnetic field, shielding currents with the critical current density $J_{\mathrm{c}}$ are obtained. If there is no electric field by shielding effect, situation of the currents is not changed. Though the critical current density $J_{\mathrm{c}}$ has a dependence on the magnetic field, the Bean model $\left(J_{\mathrm{c}}=\right.$ constant $)$ is applied to the present analysis since the critical current density is almost constant in the low cryogenic temperature.

To implement the critical state model (4) into the T-method in (1), we use the following artificial conductivity scheme $[5,6]$. At first, conductivity in all elements is set to very large 


\section{Physica $C$}

value, e.g. $10^{13}[1 / \Omega \mathrm{m}]$, assuming the superconductor is a very good conductor. If current over the critical current density $J_{\mathrm{c}}$ is obtained, the conductivity of the element is corrected as follows,

$$
\begin{cases}\sigma_{\text {new }}=\sigma_{\text {old }} \frac{J_{\mathrm{c}}}{\mathrm{J}} & \text { if } \mathrm{J}>\mathrm{J}_{\mathrm{c}}, \\ \sigma_{\text {new }}=\sigma_{\text {old }} & \text { if } \mathrm{J} \leq \mathrm{J}_{\mathrm{c} .}\end{cases}
$$

In each time step of the T-method in (1), conductivity distribution is modified by (5) so that the shielding current $\mathbf{J}$ is smaller than the critical current $J_{\mathrm{c}}$ in all region of the HTS.

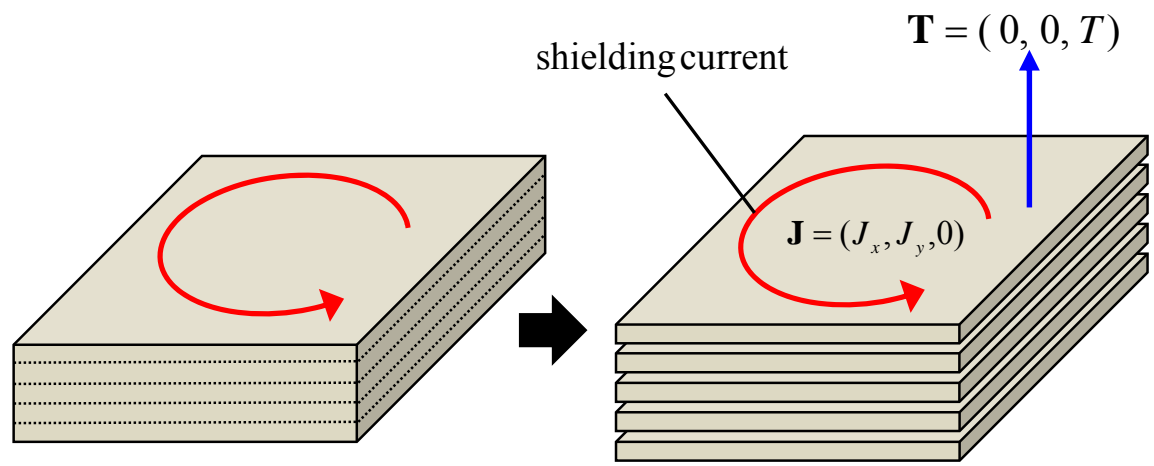

Fig. 2. Thin plate multi-layers modeling of HTS

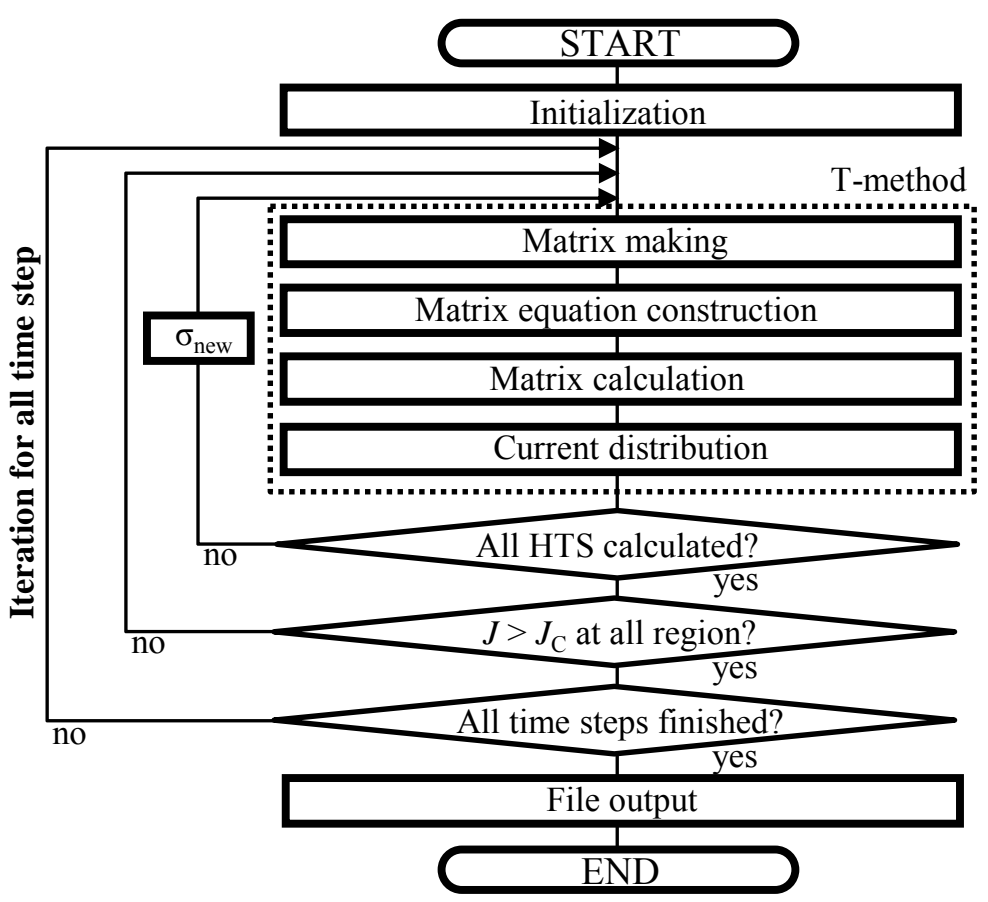

Fig. 3. Flowchart of simulation of magnetization process 
A flowchart of the simulations of the magnetization process of the Pure-type HTS undulator based on the T-method is indicated in Fig. 3. The T-method calculations of the shielding current $\mathbf{J}$ are carried out for the individual HTS and the conductivity distribution $\sigma$ is modified according to (5) in each time step. When the condition $|\mathbf{J}|<J_{\mathrm{c}}$ is satisfied in all HTSs, the simulation proceeds to the next time step. This calculation is repeated from the initial time with $\mathbf{B}_{\mathbf{0}}=B_{\max }$ to the final time with $\mathbf{B}_{\mathbf{0}}=B_{\min }$ (Fig.1). We carry out numerical discretization of the Pure-type HTS undulator by uniform cubic grids and solve the discretized equation of (1) by using three-dimensional finite differential method in time domain.

\section{Numerical examples}

The first numerical example is three HTSs undulator indicated in Fig. 1. The size of individual HTS is taken to be $10 \mathrm{~mm} \times 15 \mathrm{~mm} \times 4 \mathrm{~mm}$. The external magnetic field $\mathbf{B}_{0}=\left(0,0, B_{0}\right)$ is reduced from the initial value $B_{\max }=2.0 \mathrm{~T}$ to the final value $B_{\min }=-0.6 \mathrm{~T}$ linearly during 13.0 seconds, which means that $\partial B_{z} / \partial_{t}=-0.2[\mathrm{~T} / \mathrm{sec}]$ is imposed in the inhomogeneous term of (1). Typical shielding current distributions in the magnetization process are shown in Fig. 4(a). We can observe that the shielding current penetrates into deep inside of each HTS. Figure 4(b) shows a vertical distribution of magnetic field beyond the undulator, which is calculated by using the induced shielding currents of Fig. 4(a). Figure 5 shows plots of the vertical magnetic field component along $x$-axis on $1 \mathrm{~mm}$ beyond the undulator at the moments $B_{0}=$ $1.8,1.6,1.4,1.0,0.6,0.2,-0.4$ and $-0.6 \mathrm{~T}$ respectively. We confirm that the developed numerical code sufficiently simulate the magnetization process of the Pure-type HTS undulator from comparison the simulation in Fig. 5(a) with the measurement in Fig. 5(b) [2].

For a practical use of the Pure-type HTS undulator, the sinusoidal distribution of the vertical component of the magnetic field has to oscillate by the same amplitude for both of positive and negative sides. In addition, the amplitude of the magnetic field should be stronger than 1.0T [1]. In the present analysis, two HTS modules in Fig. 1 are placed upper and lower sides as undulator for the electron trajectory. The total field of $1.0[\mathrm{~T}]$ will be almost obtained in the present system. These conditions are almost achieved by the critical current $J_{\mathrm{c}}=$ $7.0 \times 10^{8}\left(\mathrm{~A} / \mathrm{m}^{2}\right)$ and the final external magnetic field to be $B_{\min }=-0.414 \mathrm{~T}$. Finally, we apply the developed code to a larger system of ten HTSs undulator. Fig. 7(a) shows the induced shielding current distribution in the HTS and Fig. 7(b) shows the vertical component of the 
magnetic field beyond the undulator. Almost sinusoidal field is obtained in Fig. 7(b). Fig. 7(c) shows the estimated single electron trajectory with $2 \mathrm{GeV}$ energy for X-ray radiation [1], which is calculated by the Runge-Kutta method to use the magnetic field distribution. The electron moves along a wiggling trajectory owing to the magnetic field of Fig. 7(b) but the motion is distorted from a purely sinusoidal shape (see top view of Fig. 7(c)) a little bit.
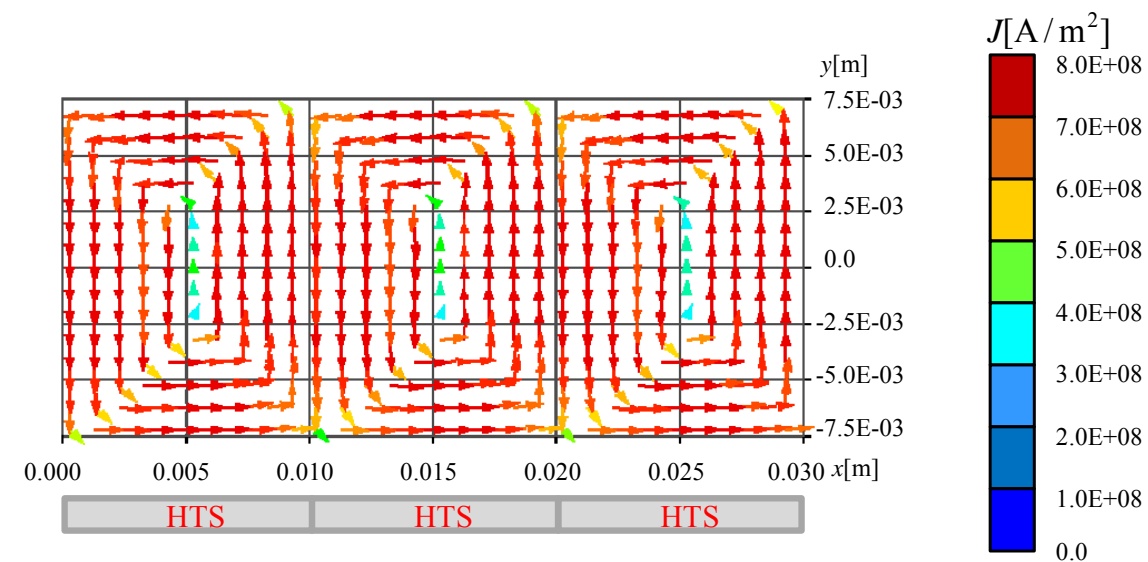

(a)

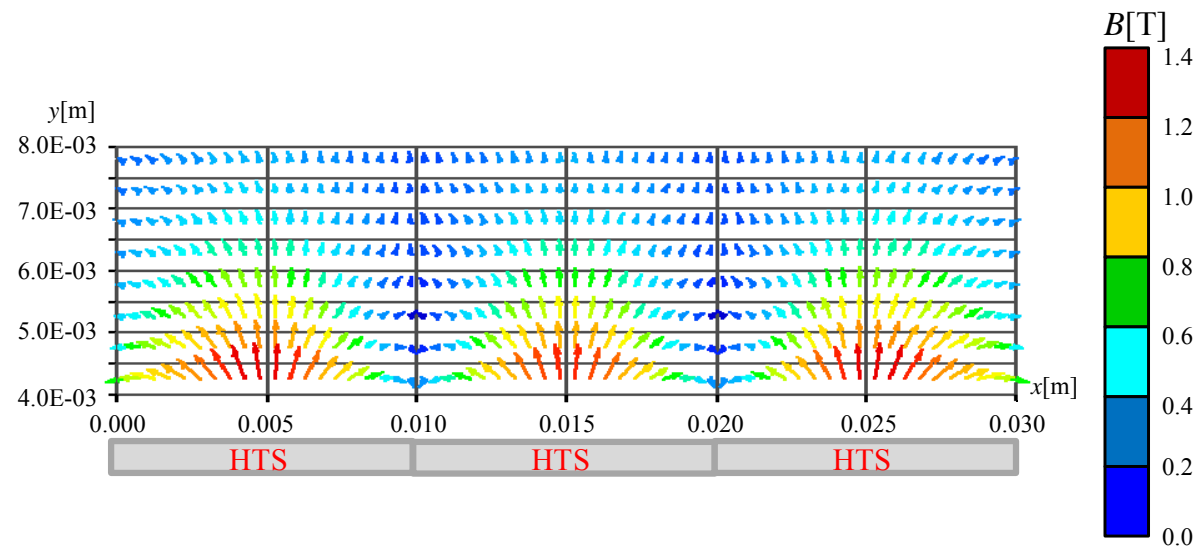

(b)

Fig. 4. Shielding current distribution on HTS (a) and magnetic field distribution in vertical plane on HTS (b) 


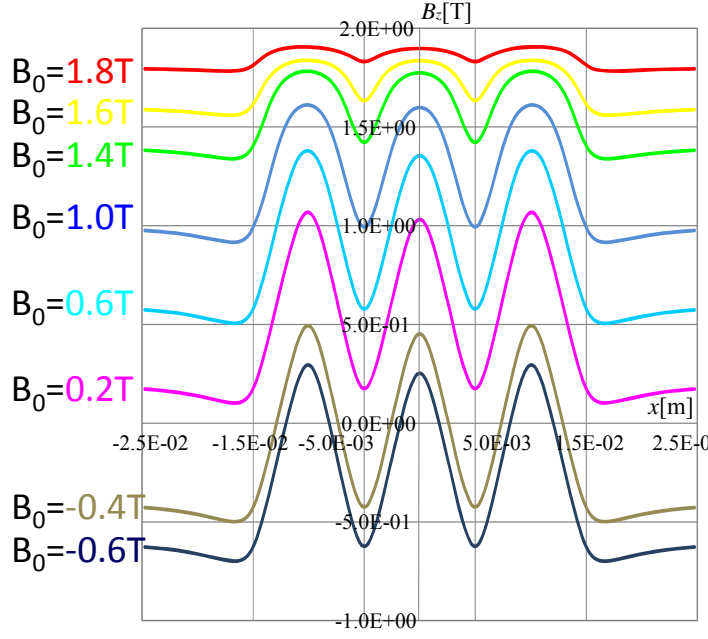

\begin{tabular}{|l|l|l|}
\hline HTS & HTS & HTS \\
\hline
\end{tabular}

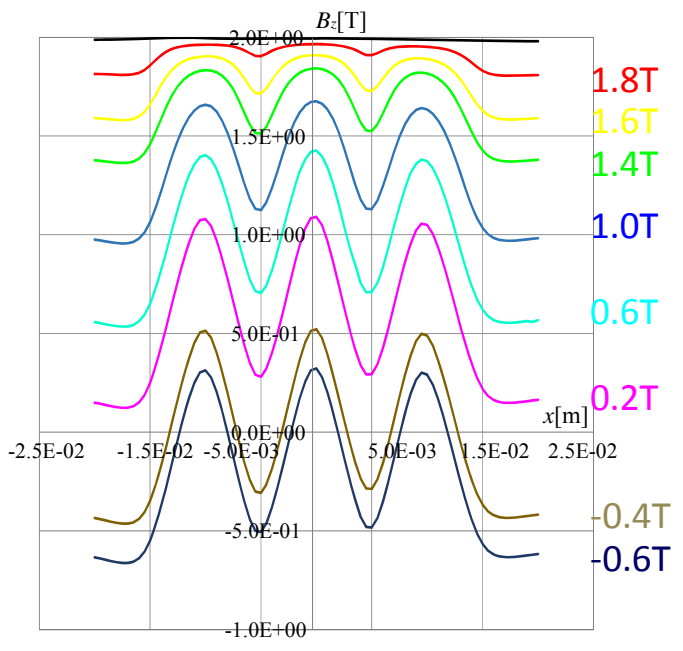

\begin{tabular}{|l|l|l|}
\hline HTS & HTS & HTS \\
\hline
\end{tabular}

(b)

Fig. 5. Magnetic field distribution beyond undulator of simulation result (a) and measurement data (b) [2] in magnetization process

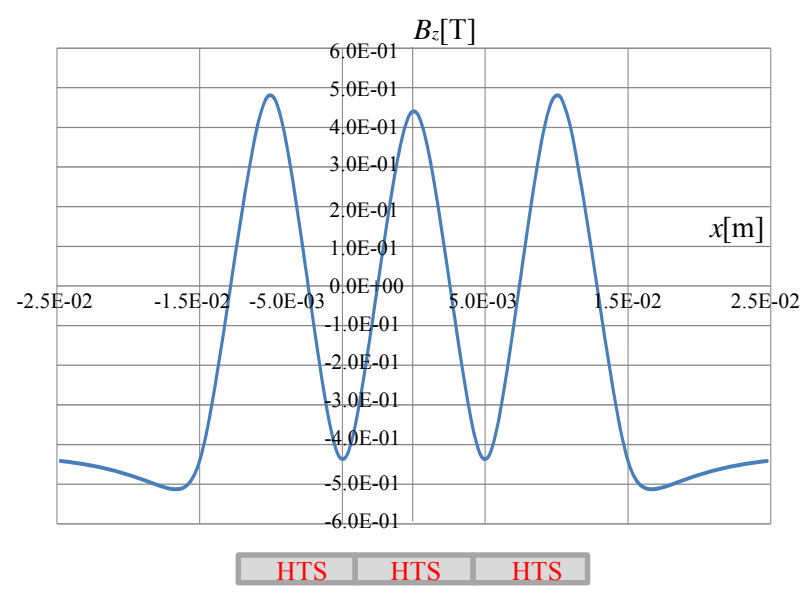

Fig. 6. Final magnetic field distribution vertical

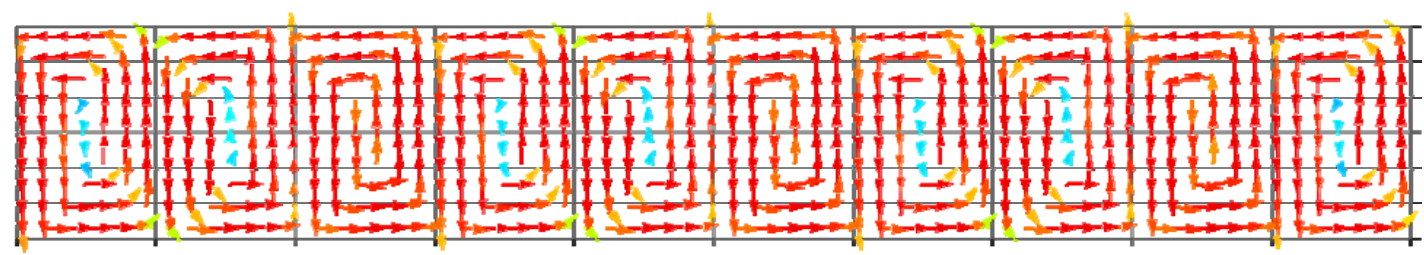

\begin{tabular}{|l|l|l|l|l|l|l|l|l|l|} 
HTS & HTS & HTS & HTS & HTS & HTS & HTS & HTS & HTS & HTS \\
\hline
\end{tabular}

(a) Shielding current distribution 


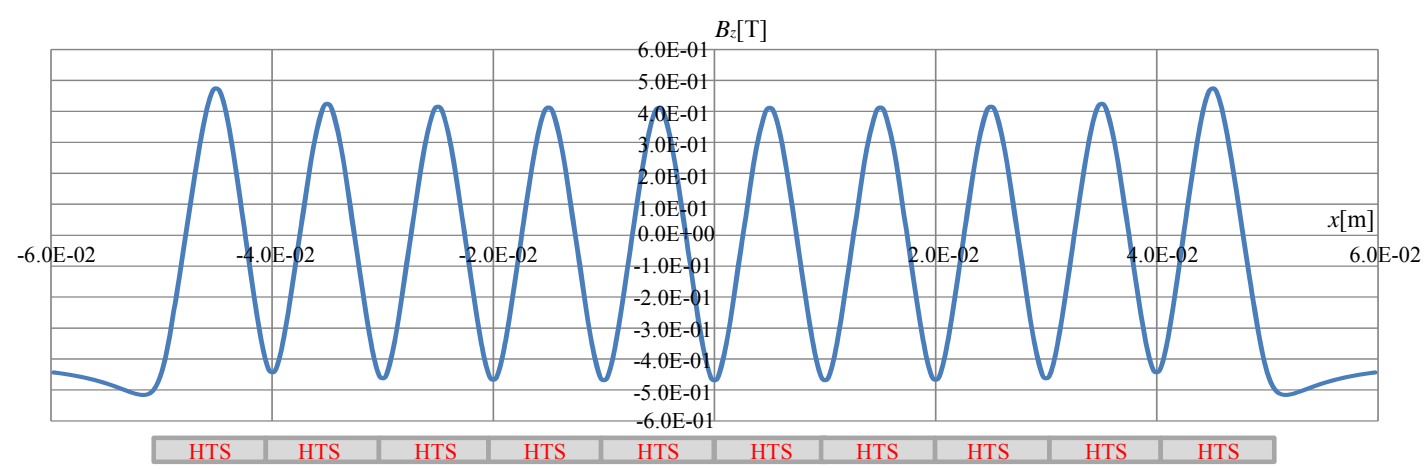

(b) Distribution of vertical component magnetic field

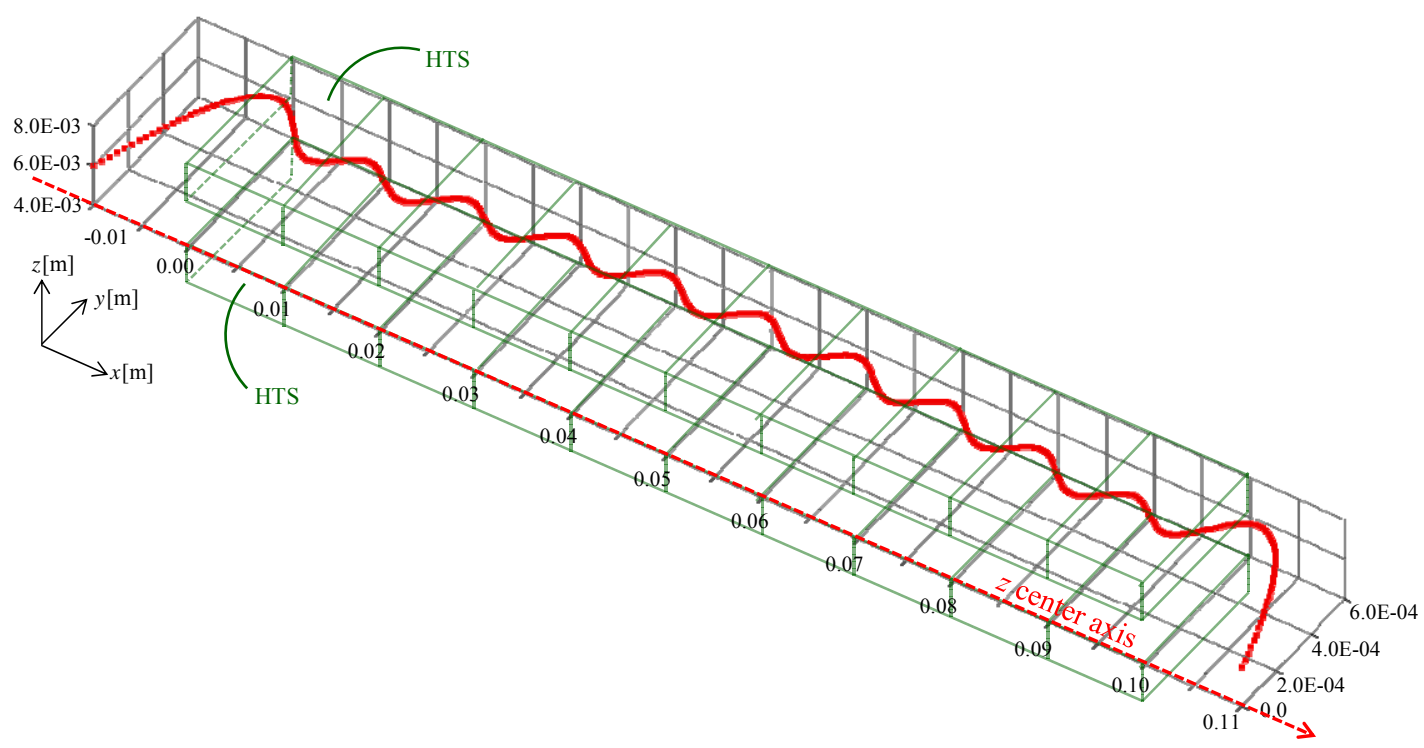

(3D view)

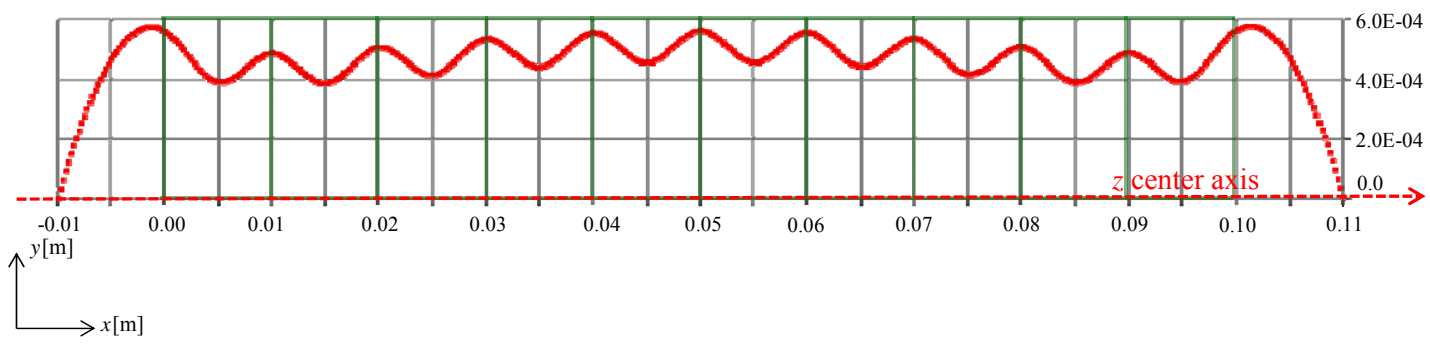

(Top view)

(c) Estimated single electron trajectory with $2 \mathrm{GeV}$ energy

Fig. 7. Simulation of ten HTSs undulator 


\section{Conclusion}

To assist the design of the HTS magnets array in the Pure-type HTS undulator, this paper has presented the development of the numerical simulation code of the HTS magnetization process based on the T-method with the critical state model. The numerical simulation is compared with experimental measurements of the magnetic field distribution in the magnetization process. The developed code sufficiently simulates the HTS magnetization process. As an example of applications of this code to the HTS undulator design, appropriate applied external magnetic field of the applied external magnetic field on the HTS was estimated. In the present analysis, the electron orbit in the undulator magnetic field calculated by the developed code is slightly distorted. This simulation result should be more extended from the viewpoint of the FEL performances and feedback to the design of HTS alignment.

\section{References}

[1] T. Kii and R. Kinjo, Application of bulk high-temperature superconductor to insertion device for synchrotron radiation, J. Cryo. Soc. Jpn. 46 (2011) 118-124.

[2] T. Tanaka, R. Tsuru and H. Kitamura, Pure-type superconductor permanent magnetundulator, J. Synchrotron Rad. 12 (2005) 442-447.

[3] T. Tanaka, T. Hara, H. Kitamura, R. Tsuru, T. Bizen, X. Mare'chal, T. Seike, Application of high-temperature superconducting permanent magnets to synchrotron radiation sources, Physical Review Special Topics - Accelerators and Beams 7 (2004) 090704.

[4] T. Sugiura, H. Hashizume and K. Miya, Numerical electromagnetic field analysis of type-II superconductors, Int. J. Appl. Electromagn. Mater. 2 (1991) 183-196.

[5] M. Tsuchimoto, K. Demachi and I. Itoh, Numerical evaluation of uniform magnetic field within superconducting Swiss roll, Physica C 412-414 (2004) 719-722.

[6] M. Tsuchimoto, Numerical Evaluation of Field Profile in an Undulator with Bulk HTS, Physics Procedia 27 (2012) 452-455.

[7] H. Kawaguchi, S. Ito and M. Tsuchimoto, Design of HTS Undulator Magnet Array Based on Simulation of Magnetization Process, Proceedings of the International Conference on Simulation Technology (JSST 2013) OS13-6 (2013). 